\title{
La gestión estratégica empresarial desde las tecnologías de la información y la comunicación. Puntos y reflexiones.
}

\section{Strategic business management from Information and Communication \\ Technologies: Points and Reflections.}

1 Marco Antonio Gavilanes Sagñay

https://orcid.org/0000-0002-7470-3732

Escuela Superior Politécnica de Chimborazo (ESPOCH)", Facultad de Administración de Empresas, Riobamba, Chimborazo, Ecuador

marco.gavilanes@espoch.edu.ec

2 Fredy Gavilanes Sagnay

https://orcid.org/0000-0001-5494-374X

Departamento de Ciencias de la Computación, Universidad de las Fuerzas Armadas ESPE sede Santo Domingo, Santo Domingo, Ecuador,

fmgavilanes1@espe.edu.ec

Departamento de Informática y Ciencias de la Computación, Facultad de Ingeniería de Sistemas, Escuela Politécnica Nacional, Quito, Ecuador

fredy.gavilanes@epn.edu.ec

3 Nathalie Azucena Chávez Granados (iD) https://orcid.org/0000-0002-4764-9160 Investigador Independiente, Riobamba, Chimborazo, Ecuador nathaliechavezg@gmail.com

Artículo de Investigación Científica y Tecnológica Enviado: 09/11/2021

Revisado: $24 / 11 / 2021$

Aceptado: 14/12/2021

Publicado:05/01/2022

DOI: $\underline{\text { https://doi.org/10.33262/ap.v4i1.130 }}$
Cítese: gestión estratégica empresarial desde las tecnologías de la información y la Cítese: comunicación. Puntos y reflexiones. AlfaPublicaciones, 4(1), 46-61. https://doi.org/10.33262/ap.v4i1.130

ALFA PUBLICACIONES, es una Revista Multidisciplinar, Trimestral, que se publicará en soporte electrónico tiene como misión contribuir a la formación de profesionales competentes con visión humanística y crítica que sean capaces de exponer sus resultados investigativos y científicos en la misma medida que se promueva mediante su intervención cambios positivos en la sociedad. https://alfapublicaciones.com 


\section{Palabras}

claves: Gestión

estratégica,

Tecnologías de

la Información

y la

Comunicación,

Organizaciones

empresariales,

factores

determinantes.
Keywords:

Strategic

management,

Information

and
Resumen

Introducción. El papel de la gerencia estratégica en las organizaciones empresariales es de gran importancia, unido a la necesidad de tener una eficiente administración de los recursos, una logística estructurada con un personal de elevada calificación, innovador, con alto grado de competitividad para incorporar la utilización de las TICs, en su actividad diaria. Objetivo. Determinar los aspectos involucrados en la gestión estratégica empresarial desde la incorporación de las TICs, los factores determinantes y los desafíos que conlleva. Metodología. El estudio utilizó una metodología descriptiva, no experimental, basada en métodos teóricos y la revisión documental, permitiendo establecer los argumentos esenciales relacionados con el tema. Los criterios establecidos tales como: el papel de la gerencia estratégica en las organizaciones empresariales, los elementos del proceso gerencial, los desafíos de la incorporación de las TICs y los factores determinantes para la adopción de las TIC conformaron las ideas centrales de la investigación. Resultados. 1. Los estudios realizados demuestran que el liderazgo y la innovación constituyen factores concluyentes de supervivencia de las empresas. 2. Reafirma que el uso de las TICs constituye una herramienta fundamental e imprescindible para perfeccionar la Gestión estratégica. 3. Las Mipymes, tienen una inherente fragilidad por la falta de recursos, dado los continuos y acelerados cambios tecnológicos y de mercado. 4. Se precisó la importancia de los factores determinantes en la adopción de las TICs. 5. La utilización de las TICs debe formar parte del plan de negocios y corresponderse con la estrategia corporativa y organizativa de la misma. Conclusiones. La revisión sistemática de la literatura permitió confirmar la importancia de la incorporación de las TICs como herramienta fundamental para la mejora de la Gestión estratégica de las empresas. Se aportan elementos técnicos y organizacionales, para proceder a este proceso, en función de las características de cada organización empresarial.

\section{Abstract}

Introduction. The role of strategic management in business organizations is of great importance, together with the need to have an efficient administration of resources, structured logistics with highly qualified, innovative personnel, with a high degree of 
Communicatio

n Technologies,

Business

organizations, determining

factors. competitiveness to incorporate the use of ICTs, in their daily activity. Target. Determine the aspects involved in strategic business management from the incorporation of ICTs, the determining factors and the challenges involved. Methodology. The study used a descriptive, non-experimental methodology, based on theoretical methods and documentary review, allowing the essential arguments related to the subject to be established. The established criteria such as: the role of strategic management in business organizations, the elements of the managerial process, the challenges of incorporating ICTs and the determining factors for the adoption of ICTs formed the central ideas of the research. Results. 1. The studies carried out show that leadership and innovation are conclusive factors for the survival of companies. 2. Reaffirms that the use of ICTs is a fundamental and essential tool for perfecting Strategic Management. 3. Mipymes have an inherent fragility due to lack of resources, given the continuous and accelerated technological and market changes. 4. The importance of the determining factors in the adoption of ICTs was specified. 5. The use of ICTs must be part of the business plan and correspond to the corporate and organizational strategy of the same. Conclusions. The systematic review of the literature confirmed the importance of incorporating ICTs as a fundamental tool for improving the strategic management of companies. Technical and organizational elements are provided to proceed with this process, depending on the characteristics of each business organization.

\section{Introducción}

En la actualidad cualquier empresa requiere necesariamente para realizar su normal funcionamiento, de la interrelación de un conjunto de acciones de carácter gerencial; dado las tendencias que caracterizan a un mundo globalizado que se asienta en el desarrollo de los mercados con un nivel alto de exigencias, donde tienen cabida solo aquellas empresas que son capaces de tener un elevado sentido de constante transformación, acorde a lo que dictan dichos mercados. Es precisamente este carácter gerencial, una de las características de la gestión estratégica de una empresa. 
Estas transformaciones que se requieren para las empresas se traduce en el necesario dinamismo que deben tener para adecuarse a los continuos cambios y desafíos que exige el mercado, constituyendo esta otra de las características de la época actual, que de acuerdo a Roman, De Pelekais, \& Pelekais,(2009), solo pueden ser impulsados por organizaciones empresariales y funcionarios gerenciales que aplican una política de constante revisión, que profesen la adecuación e innovación de los procesos administrativos y gerenciales que administran y lideran.

En esta dirección, gran importancia reviste el papel de la gerencia estratégica en las organizaciones empresariales, dado que las mismas deben forzosamente que cumplir con los objetivos propuestos, lo cual según Marín, Ruiz, \& Henao, (2016), debe realizarse mediante una adecuada y eficiente administración y conducción efectiva de los recursos, los que deberán ser dirigidos en la óptima planificación estratégica, en el aumento de capital, en la adquisición y uso de tecnología de punta, en la logística estructurada en función a las necesidades de la organización empresarial y en la incorporación de personal con elevada calificación, innovador y con elevado grado de competitividad.

Los autores e investigadores que actualmente realizan sus trabajos relacionados con la organización y óptimo funcionamiento de las empresas, entre los que citamos a Panchi, Casquete, \& Rivadeneira. (2019), señalan que centran los mismos, entre otros aspectos, esencialmente en dar respuesta a las problemáticas relacionadas a cómo las organizaciones deben enfrentar los cambios que se generan en sus entornos, así como a detectar los factores que les permitirá continuar siendo competitivas.

En este sentido autores como Turbay-Posada (2013), son del criterio que el liderazgo y la innovación constituyen factores concluyentes en la supervivencia de las organizaciones empresariales.

No obstante, estos factores esenciales en nuestros tiempos en una organización empresarial, y de acuerdo con autores como Rueda (2013), solo es posible lograrlo a través de una gestión gerencial enfocada en la concepción de innovación continua. Lo anterior conlleva a la creación de un direccionamiento estratégico categórico y que a su vez permita se destinen recursos económicos, técnicos y humanos adecuados con vistas a lograr soluciones transformadoras, que ofrezcan respuestas a las demandas internas y externas que debe asumir de manera constante una organización empresarial competitiva.

En relación a ello, esencial importancia reviste el conocimiento de los elementos más importantes que conforman el proceso gerencial propiamente, que, a decir de Chiavenato (1999), lo constituyen: La planeación, la organización, la dirección y el control del proceso. Entendiendo según el autor de referencia lo siguiente: 
- La planeación, es la acción que tiene como significado establecer y estudiar de manera anticipada los objetivos y acciones, así como en ella están contenidos los métodos, procedimientos y planes de la organización, así como define los procedimientos adecuados para alcanzar dichos objetivos. También incluye las diversas tareas que deben ser ejecutadas para conseguir los logros de la organización empresarial, trazar como deben ser ejecutadas y precisar en qué momento deben realizarse.

- La Organización, es el proceso de organización, de integración, de estructuración de los recursos disponibles y los órganos encargados de la administración y del establecimiento de las relaciones entre ellos con la finalidad de estipular las atribuciones que le corresponde a cada uno.

- La Dirección, es la que se encarga de poner en operaciones los lineamientos que fueron establecidos durante la planeación y que posteriormente en la fase de organización fueron estructurados. Es el encargado de suministrar el dinamismo y la operatividad al proceso gerencial, por lo que exige de una habilidad e innovación gerencial, dado que en esta fase se guían, motivan, lideran y administran las habilidades y los esfuerzos del capital intelectual de la empresa, para lograr alcanzar los objetivos organizacionales propuestos.

- El Control, tiene como propósito crear la garantía de que los resultados de lo que fue planeado, organizado y dirigido, se justen cuanto más posible a los objetivos preestablecidos. Autores como Luzardo y Peleakais (2007) expresan que su particularidad reside en la verificación de si la actividad que se controla se encuentra alcanzando o no los objetivos o resultados esperados. En este sentido, autores como Marín, Ruiz, \& Henao, (2016), han expresado que una óptima comunicación y motivación de los directivos y del personal de una organización empresarial, representa la clave para determinar un problema dado, para obtener mejores desempeños y elementos de control, así como, para incrementar la productividad, obteniendo más utilidades y una economía y eficiencia mayor.

El desarrollo tecnológico alcanzado en los últimos años y en especial aquellos comprendidos en el desarrollo de las Tecnologías de la Información y las Comunicaciones (TICs), han ofrecido diferentes herramientas que le han posibilitado a la gerencia estratégica de las organizaciones empresariales, desempeñar una mejor función de dirección, entre los que podemos mencionar a los sistemas de información integrados, la búsqueda de datos y la aplicación de sistemas inteligentes entre otros.

En relación a ello, Castellanos (2003) indica que la gestión de las organizaciones empresariales, en los últimos tiempos, de manera creciente, han aumentado la dependencia del rol y la interpretación de la tecnología y la comunicación interna de las 
organizaciones, por lo que, en este sentido, se supone que el uso de las TICs constituye un factor concluyente para una adecuada y óptima gestión empresarial. En correspondencia con lo señalado la investigación que se presenta tiene como objetivo: Determinar los aspectos involucrados en la gestión estratégica empresarial desde la incorporación de las TICs, los factores determinantes y los desafíos que conlleva.

\section{Beneficios de la incorporación de las TICs a las organizaciones empresariales}

Diferentes autores han señalado los beneficios que incorporan las TICs a las organizaciones empresariales, entre ellos citamos a Buenrostro y Hernández (2019), quienes destacan que el uso de las mismas, se considera como un aspecto que trae consigo un aumento de la competitividad mediante un aumento de la productividad, de la eficiencia y rentabilidad de la inversión que realiza. No obstante, plantean que el resultado que de ello se deriva dependerá de las características propias de la tecnología que se utilice, además de las capacidades internas de estas organizaciones.

Las incorporaciones de las TICs a las empresas brindan cuantiosos beneficios para su propio desarrollo en lo que a eficiencia, desarrollo integral y competitividad se refiere, en este sentido Guillén (2007), Sheel y Rivera (2009) han explicado que la inclusión de las TICs en las empresas posibilita la modernización y la consiguiente agilización de los procesos de la Empresa, provocando un incremento de la productividad y competitividad, en un mercado que de manera continuada se globaliza, lo que implica que el diseño y operación que realizan actualmente las mismas, son dependiente significativamente del uso de las TICs, siempre y cuando, sean utilizadas de manera adecuada y dinámica con la finalidad de lograr óptimos niveles de desempeño.

En este sentido investigaciones realizadas por Rivas y Stumpo, (2013), exponen que en las últimas décadas la incorporación de las TICs a las organizaciones empresariales ha contribuido al incremento de la economía de los países y según Consoli (2012) ha actuado de manera positiva tanto en los aspectos que se relacionan con la intensidad del capital y la ampliación del nivel de automatización en las mismas, que ha implicado mayor productividad, eficiencia, porcentajes de mercado y rentabilidad, sino también, de acuerdo con Cepal (2013), en aspectos que se vinculan de manera indirecta con la utilización de la tecnología, con los referidos a los cambios organizacionales y de igual forma, con los procesos y utilización de la información y comunicación y el desarrollo de nuevas modalidades que acceden al conocimiento.

\section{Desafíos de las organizaciones empresariales frente al escenario de incorporación de las TICs.}

Para desempeñarse actualmente en el entorno de negocios, que es complejo y muy competitivo, las organizaciones empresariales requieren tener, de acuerdo a Chenchall 
(2007), información significativa de carácter múltiple, tanto cualitativa como cuantitativa, que nutra al proceso de mejoras continuas de la gestión de la empresa, con la finalidad de lograr los objetivos organizacionales deseados.

Respecto a ello Jones, Motta y Alderete (2016), explican que para lograr lo anterior, las empresas precisan de diferentes indicadores que les permite medir su comportamiento organizacional, los que pueden alcanzar un determinado grado de complementación entre ellos que les posibilite conseguir una visión integradora de la organización.

Las grandes empresas se encuentran preparadas para adaptarse y transformarse de manera continua al entorno actual, no obstante, en el caso de las micro, pequeñas y medianas empresas (Mipymes), sobre las cuales centraremos nuestro artículo, su inherente fragilidad por la falta de recursos se vuelve más crítica por los acelerados cambios tecnológicos y de mercado.

Al respecto investigaciones realizadas por Dewan y Riggins (2005), enuncian que las Mipymes cuentan con desventajas significativas en relación a las grandes empresas, y en concordancia con ello, Consoli, (2012), reiteran que ello es debido a que se encuentran atadas a limitantes económicos, técnicos y humanos que les impide incorporarse de forma eficiente a la tecnología.

En relación a lo explicado y de acuerdo a, Jones, Motta y Alderete (2016), debido a que la mayoría de los objetivos organizacionales están potenciados por las TICs, las Mipymes, requieren evaluar los riesgos y oportunidades propias de las mismas, por lo que necesitan además de la implementación de mecanismos adecuados para poder proceder con su uso y gestión, de la evaluación de los impactos que genera en el desempeño organizacional. Es por ello que autores como Albarracín et al., (2014), subrayan la influencia positiva que presentan las TICs sobre el rendimiento de las organizaciones empresariales.

Teniendo en cuenta lo expresado anteriormente, se debe prestar especial atención por los grupos gerenciales de las empresas a estos aspectos, de manera que puedan dirigir de manera eficiente y eficaz su gestión estratégica. Al respecto, diversos autores han alertado al respecto. Raymond, Croteau y Bergeron, (2011), afirman que es esencial que las Mipymes cuenten con el conocimiento necesario que les permita conocer cómo y en qué grado sus inversiones en TICs, pudieran acompañarse con prácticas de utilización y gestión que favorezcan un óptimo aprovechamiento de los beneficios que se asocian a estas tecnologías.

En referencia con lo abordado, Girgin, Kurt and Odabasi (2011), indican que las Mipymes no se encuentran de manera general realizando la explotación de las TICs como las grandes empresas. Ello se debe a que las mismas cuentan con tecnología y 
recursos limitados, aunque en este sentido, la estructura menos compleja posibilita a las empresas más pequeñas una mayor flexibilidad a los cambios que se generan.

Relacionado con lo expuesto, Consoli (2012), expresa que los factores que inhiben, o sea, que no estimulan las inversiones de las organizaciones empresariales en TIC están referidos a:

- Financiero: Representa una elevada inversión inicial y dificultad en el acceso al crédito.

- Infraestructura: Requieren de energía, de ancho de banda y conexión a Internet confiable.

- Organizacional: Presentan falta de personal calificado y estrategia Incoherente.

- Tecnológico: Presentan un desarrollo de la tecnología sin la Información adecuada.

Referente a ello, para estimular la inversión en TICs en las Mipymes es fundamental desarrollar políticas públicas, con la finalidad de eliminar la brecha digital existente, proporcionar el acceso gratuito a la internet y garantizar el apoyo a la capacitación del personal involucrado.

\section{Factores determinantes de la adopción de las TICs.}

Existen diferentes estudios y contribuciones respecto a la adopción de las TICs en las pequeñas y medianas empresas. En referencia a ello autores como Skoko et al., 2007, expresan que se pueden clasificar los factores determinantes en 5 grupos, los que se refieren a: Individual, Organizacional, Ambiental, Tecnológico y Económico.

En la siguiente figura se detallan, los factores determinantes para la adopción de las TICs en las empresas. 
Figura No.1: Factores determinantes para la adopción de las TICs por las empresas

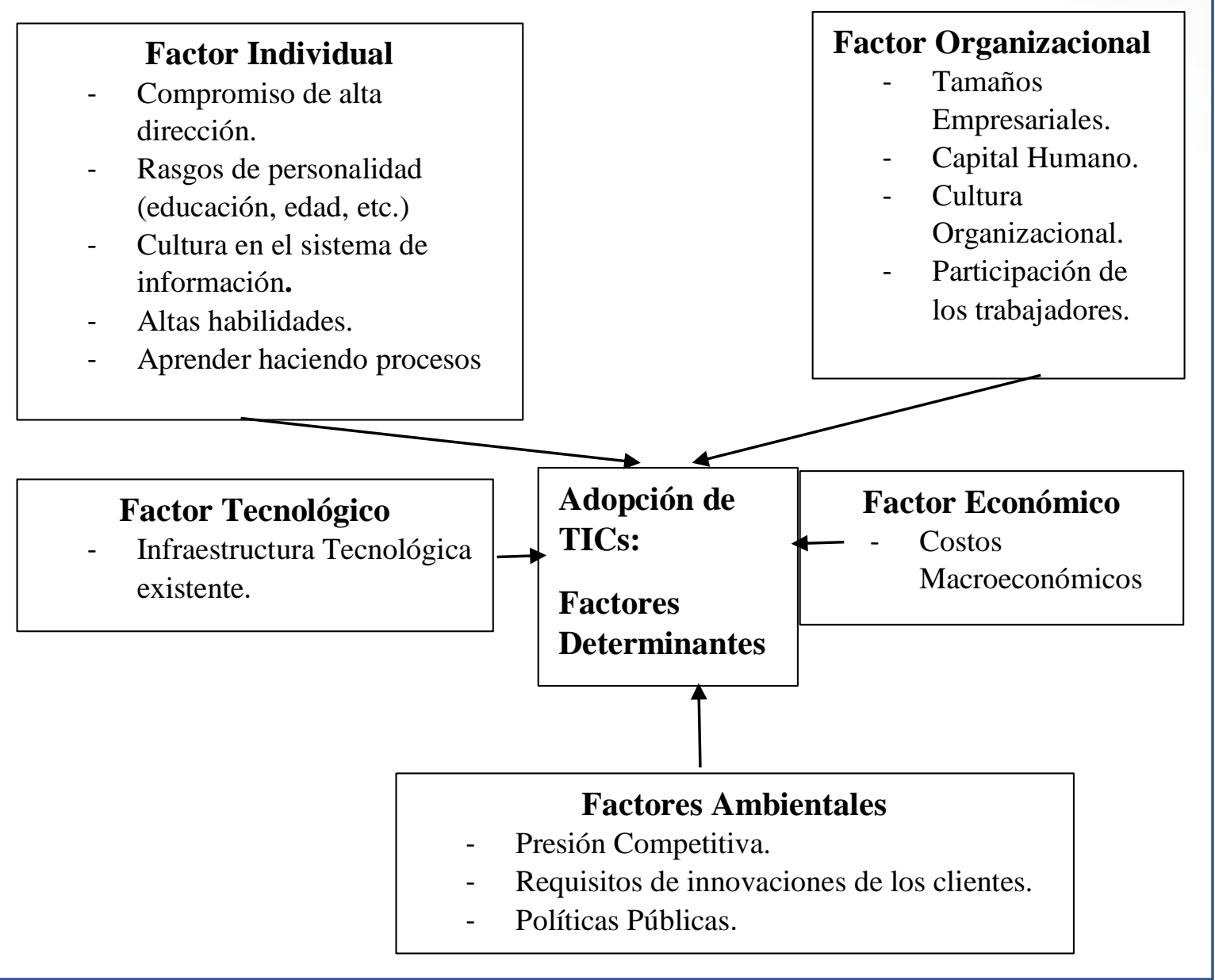

Nota: Skoko et al., 2007, modificado por Consoli, Domenico (2012).

https://www.sciencedirect.com/science/article/pii/S187704281203457X

La incorporación de las TICs en una organización empresarial no resulta de manera natural o automática, sino que presenta determinada complejidad, en función de las motivaciones existentes. Al respecto autores como Dyerson y Spinelli (2011), señalan que el proceso de adopción de las TIC se presenta complicado y el mismo se incentiva por la existencia de las siguientes condiciones:

- Condiciones de negocios: Sensibilidad y compromiso de la alta dirección Estratégica.

- Condiciones organizativas: Contar con la presencia de un puntal de TICs, ya Sea un empresario, un gerente o cualquier otra Empleado del departamento de Tecnología o Externo a la empresa (un consultor o proveedor). 
- Condiciones de implementación y manejo: Contar con un inventario de hardware, software y herramientas TIC con recursos humanos calificados.

En este contexto, los propios autores señalan que la evaluación de los factores de adopción y los impactos que provocan en las organizaciones empresariales, es fundamental para comprender como estimular en las Mipymes el proceso de incorporar inversiones en nuevas tecnologías para adquirir ventajas competitivas y buenas prácticas empresariales.

En concordancia con lo anterior, la utilización de las TICs por las empresas debe ser uno de los eslabones fundamentales de su gestión estratégica, de manera que le permita aprovechar sus características y beneficios tecnológicos en el desarrollo de todas sus áreas.

En esta dirección se han referido diversos autores, entre los que citamos a Vega et al., (2008), quienes afirman que la utilización de las TICs debe ser incorporado y detallado en un plan de negocios y estar en correspondencia con la estrategia corporativa y organizativa de la organización empresarial, de manera que posibilite la explotación al máximo de su potencial tecnológico.

Los propios autores expresan que, en este contexto, en las empresas se pueden visualizar dos situaciones extremas, o que la alta gerencia no favorece la inversión en tecnologías o que la gerencia esté muy interesada con la inversión en TICs, pero la empresa como tal, su estructura y el Plan estratégico no son adecuados. En este sentido es que las empresas deben realizar una profunda evaluación, en función de sus características, para de esa manera implementar un proceso de adopción cuanto más eficiente y eficaz.

\section{Metodología}

En el estudio se utilizó una metodología descriptiva, no experimental. Basada en métodos teóricos dentro de estos el histórico-lógico, analítico -sintético, inductivodeductivo y la revisión documental, lo cual permitió realizar la revisión de la literatura precedente y establecer los argumentos esenciales relacionados con el tema. Dentro de los temas abordados se tuvieron en cuenta: El papel de la gerencia estratégica en las organizaciones empresariales, los elementos del proceso gerencial, los desafíos de la incorporación de las TICs a las empresas y los factores determinantes para la adopción de las TICs por las empresas, entre otros, favorecieron la calidad del trabajo realizado.

\section{Resultados y Discusión}

El análisis de los referentes teóricos consultados nos permite establecer cinco resultados claves, los cuales constituyen los puntos básicos de reflexión: 
1. Los estudios realizados por Turbay-Posada (2013) han demostrado que el liderazgo y la innovación constituyen factores concluyentes en la supervivencia de las organizaciones empresariales y ello en concordancia con Rueda (2013), solo es posible lograrlo mediante una gestión gerencial enfocada en la concepción de innovación continua, conlleve a la creación de un direccionamiento estratégico categórico, que permita se destinen recursos económicos, técnicos y humanos adecuados con vistas a lograr soluciones transformadoras, que ofrezcan respuestas a las demandas internas y externas que debe asumir de manera constante una organización empresarial competitiva.

2. La revisión sistemática de la literatura permite reafirmar que en el contexto actual el uso de las Tecnologías de la Información y la Comunicación, constituye una herramienta fundamental e imprescindible para perfeccionar la Gestión estratégica de las instituciones empresariales. El desarrollo tecnológico alcanzado en los últimos años, y en especial aquellos comprendidos en el desarrollo de las TICs, han ofrecido diferentes herramientas que le han posibilitado a la gerencia estratégica de las organizaciones empresariales, desempeñar una mejor función de dirección, entre los que podemos mencionar a los sistemas de información integrados, la búsqueda de datos y la aplicación de sistemas inteligentes entre otros. En este sentido Castellanos (2003,) afirman que el uso de las TICs, constituyen un factor concluyente para una adecuada y óptima gestión empresarial.

3. Las investigaciones realizadas por diversos autores, tales como Dewan y Riggins (2005), expresan que las Micro, pequeñas y Medianas Empresas (Mipymes), en relación con las grandes empresas, tienen una inherente fragilidad por la falta de recursos, lo cual se vuelve más crítica por los continuos y acelerados cambios tecnológicos y de mercado, lo que hace que en concordancia con ello, Consoli, (2012), reitera que ello es debido a que se encuentran atadas a limitantes económicos, técnicos y humanos que les impide incorporarse de forma eficiente a la tecnología.

4. Se ha determinado la importancia de los factores determinantes en la adopción de las TIC en las organizaciones empresariales. En referencia a ello autores como Skoko et al., (2007), expresan que se pueden clasificar los factores determinantes en 5 grupos, los que se refieren a: Individual, Organizacional, ambiental, Tecnológico y Económico. De igual manera señalan que la evaluación de los factores de adopción y los impactos que provocan los mismos en las organizaciones empresariales, es fundamental para comprender como estimular en las Mipymes el proceso de incorporar inversiones en nuevas tecnologías para adquirir ventajas competitivas y buenas prácticas empresariales. 
5. Se ha logrado aseverar que la utilización de las TICs debe ser incorporada de forma detallada en un plan de negocios y estar en correspondencia con la estrategia corporativa y organizativa de la organización empresarial, de manera que posibilite la explotación al máximo de su potencial tecnológico, por lo que las empresas deben realizar una profunda evaluación organizacional, en función de sus características, para de esa manera implementar un proceso de adopción cuanto más eficiente y eficaz. (Vega et al., 2008),

Todo lo anterior nos sitúa en la necesidad de reflexionar sobre la trascendental importancia que tiene actualmente el uso de las TICs en el contexto empresarial, destacando en ello la necesidad de contar con un personal capacitado que logre progresivamente mejorar los resultados de su gestión mediante el uso de las Tecnologías de la Información y la Comunicación y de esta forma poder incidir positivamente en la Gestión estratégica de la empresa o entidad. Ello permitió determinar los aspectos involucrados en la gestión estratégica empresarial desde la incorporación de las TICs, los factores determinantes y los desafíos que conlleva.

\section{Conclusiones}

- La revisión sistemática de la literatura permitió confirmar la importancia de la incorporación de las Tecnologías de la Información y la Comunicación, como herramienta fundamental para la mejora de la Gestión estratégica de las organizaciones empresariales. Quedando confirmado que la utilización de las TICs por las empresas debe ser uno de los eslabones fundamentales de su gestión estratégica, de manera que le permita aprovechar sus características y beneficios tecnológicos en el desarrollo de todas sus áreas.

- En el artículo se detallan los diferentes aspectos que conllevan a la incorporación de las TICs en las organizaciones empresariales, aportando elementos técnicos y organizacionales que deben ser tenidos en cuenta por las empresas, principalmente las Mipymes, para que puedan proceder a este proceso, que de por sí se torna complejo, en función de las características de cada organización empresarial, ayudándolas a que puedan enfocarse en la optimización del desarrollo de las competencias y manejo de la las TICs, con vista al perfeccionamiento de la Gestión Estratégica de las empresas.

\section{Referencias Bibliográficas}

Albarracín J, Erazo S, Palacios F (2014). Influencia de las tecnologías de la información y comunicación en el rendimiento de las micro, pequeñas y medianas empresas colombianas. Estudios Gerenciales. Volumen 30 No.133, pp. 364-365. DOI: https://doi.org/10.1016/j.estger.2014.06.006. 
https://www.icesi.edu.co/revistas/index.php/estudios_gerenciales/article/view/18 88

Buenrostro H y Hernández M (2019). La incorporación de las TIC en las empresas. Factores de la brecha digital en las Mipymes de Aguascalientes. Econ: teor. práct no.50 México ene./jun. 2019. Economía: teoría y práctica. versión Online ISSN 2448-7481versión impresa ISSN 0188-3380.

http://www.scielo.org.mx/scielo.php?script=sci_arttext\&pid=S0188$33802019000100101 \#$ aff2.

Castellanos, O. (2003). Gestión en tecnología: Aproximación conceptual y perspectivas de desarrollo. INNOVAR 197-212. https://www.researchgate.net/publication/237040603_Gestion_en_tecnologia_A proximacion_conceptual_y_perspectivas_de_desarrollo

Comisión Económica para América Latina (Cepal) (2013), Economía digital para el cambio estructural y la igualdad, Santiago, $130 \mathrm{pp}$

Consoli, Domenico (2012), "Literature Analysis on Determinants Factors and the Impact of ICT in SMEs", Procedia-Social and Behavioral Sciences, Volume 62 (octubre), pp. 93-97. https://www.sciencedirect.com/science/article/pii/S187704281203457X.

Chenhall, R. H. (2007). Theorising contingencies in management control systems research. In C. S. Chapman, A. G. Hopwood, \& M. D. Shields (Eds.), Handbook of Management Accounting Research: Volume 1 (First ed., pp. 163 - 205). Elsevier.

ISBN (print) 9780080445649. https://research.monash.edu/en/publications/theorising-contingencies-inmanagement-control-systems-research

Chiavenato I, (1999). Administración de Recursos Humanos.

Quinta Edición - Noviembre de 1999 - Editorial Mc Graw Hill. https://www.ucipfg.com/Repositorio/MAES/MAES-08/UNIDADES-

APRENDIZAJE/Administracion\%20de\%201os\%20recursos\%20humanos(\%20le ct\%202)\%20CHIAVENATO.pdf

Dyerson, R. y Spinelli R. (2011). Equilibrio del crecimiento: un marco conceptual para evaluar la preparación para las TIC en las PYME. revista internacional de Mercadeo en Línea, vol. 1(2), págs. 43-56. DOI: 10.4018/ijom.2011040103. https://www.semanticscholar.org/paper/Balancing-Growth\%3A-A-ConceptualFramework-for-ICT-in-DyersonSpinelli/e7d09dbc0b32106db4146b38ab43acdce4593758 
Guillén, T. (2007). Las TIC en la estrategia empresarial. Anetcom. https://datos.portaldelcomerciante.com/userfiles/167/Biblioteca/93d0cb62098a0 ea3055eLaTICenlaestrategiaempresarial.pdf

Jones C, Motta J y Alderete M (2016). Gestión estratégica de tecnologías de información y comunicación y adopción del comercio electrónico en Mipymes de Córdoba, Argentina. Estudios Gerenciales. Volume 32, Issue 138, JanuaryMarch 2016, pages 4-13. https://doi.org/10.1016/j.estger.2015.12.003. https://www.sciencedirect.com/science/article/pii/S0123592316000048

Luzardo O, Pelakais C (2007). Gerencia del control interno del efectivo en los institutos y colegios universitarios públicos. https://www.researchgate.net/publication/277711685_Gerencia_del_control_inte rno_del_efectivo_en_los_institutos_y_colegios_universitarios_publicos

Marín, K. O., Ruiz, A., \& Henao, E. (2016). La motivación como un factor clave de éxito en las organizaciones modernas. Colección Académica de Ciencias Sociales, ISSN -e: 2422-0477 Vol. 3 No. 23(2), 22-35. file:///C:/Users/Usuario/Downloads/4484-Texto\%20del\%20art\%C3\%ADculo8203-1-10-20200930.pdf

Panchi Castro, N. G., Casquete Baidal, N. E., \& Rivadeneira Pacheco, J. L. (2019). La gerencia empresarial como la clave para el éxito. E-IDEA Journal of Business Sciences, 1(2), 43-49. Recuperado a partir de https://revista.estudioidea.org/ojs/index.php/eidea/article/view/7

Raymond L, Bergeron F y Croteau A. (2013). Innovation capability and performance of manufacturing SMEs: The paradoxical effect of IT integration

Journal of Organizational Computing and Electronic Commerce, 23 (3) (2013), pp. 249272.

DOI:10.1080/10919392.2013.807714. https://www.researchgate.net/publication/263731217_Innovation_Capability_an d_Performance_Of_Manufacturing_SMEs_The_Paradoxical_Effect_of_IT_Inte gration

Riggins, Frederick J and Dewan, Sanjeev (2005), "The Digital Divide: Current and Future Research Directions", Journal of the Association for Information Systems, 6 (12), pp. 298-337. DOI: 10.17705/1jais.00074.

Rivas, Diego y Stumpo, Giovanni (2013), "Las TIC en el tejido productivo de América Latina", en Novick, Marta y Rotondo, Sebastian, El desafio de las TIC en 
Argentina. Crear capacidades para la generación de empleo, Cepal, Santiago, pp. 43-78, 290

Roman, W., De Pelekais, C., \& Pelekais, E. (2009). Innovación en el proceso organizacional-clave del éxito gerencia. COEPTUM, 1(1), 62-79. ISSN: 18569706. Volumen1. Edición $\quad$ No 1. http://ojs.urbe.edu/index.php/coeptum/article/view/1626/1571

Rueda, J. F. (2013). La innovación como eje del desarrollo empresarial. Gestión \& Sociedad, 6(1), 139-146. https://www.researchgate.net/profile/Javier-RuedaGalvis/publication/263426017_Innovation_as_the_Axis_of_Business_Develop ment_La_innovacion_como_eje_del_desarrollo_empresarial/links/02e7e53acb0 ac933a5000000/Innovation-as-the-Axis-of-Business-Development-Lainnovacion-como-eje-del-desarrollo-empresarial.pdf

Sheel C y Rivera A (2009). Utilización de las TIC y su impacto en la competitividad de las empresas latinoamericana. file:///C:/Users/User/Downloads/1070Texto\%20del\%20art\%C3\%ADculo-3895-1-10-20100522.pdf.

http://revistas.urosario.edu.co/. Obtenido de Utilización de las TICs y su impacto:

http://revistas.urosario.edu.co/index.php/empresa/article/view/1070/968

Skoko H., Buerki, L. \& Ceric, A. (2007). Empirical evaluation of ICT adoption in Australian SMEs: Systemic Approach. International Conference on Information Technology and Applications, Harbin, China, IEEE, January 15-18, pp. 9-14. ISBN (electrónic) 9780980326703.https://researchoutput.csu.edu.au/en/publications/empiricalevaluation-of-ict-adoption-in-australian-smes-systemic-

Turbay-Posada, M. J. (2013). Liderazgo e innovación organizacional. vii-ix: Psicología desde el Caribe, 30(1). file:///C:/Users/Usuario/Downloads/TurbayPosadaM.J.2013.EditorialLiderazgoeInnovacionOrganizaciona.Psicologiadesdee 1CaribeVol.30No1\%20(1).pdf

Vega, A., Chiasson, M., and Brown, D. (2008). Extending the research agenda on diffusion: the case of public program interventions for the adoption of e-business systems in SMEs. Journal of Information Technology, Vol. 23, pp. 109117. https://doi.org/10.1057/palgrave.jit.2000135.

https://journals.sagepub.com/doi/10.1057/palgrave.jit.2000135.

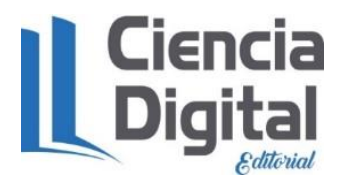


El artículo que se publica es de exclusiva responsabilidad de los autores y no necesariamente reflejan el pensamiento de la Revista Alfa Publicaciones.

\section{\Ciencia}

El artículo queda en propiedad de la revista y, por tanto, su publicación parcial y/o total en otro medio tiene que ser autorizado por el director de la Revista Alfa Publicaciones.
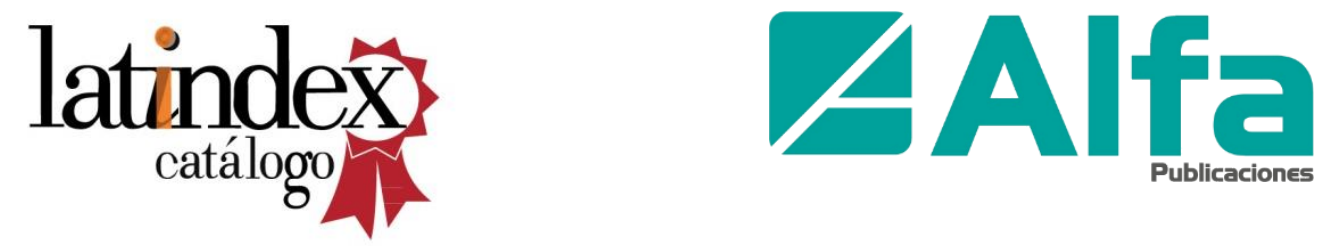

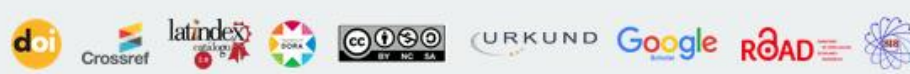
TLatinREV

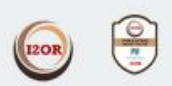

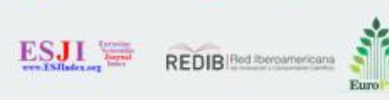

$\underbrace{}_{\text {wizdom.oi }} \overbrace{\text { OpenAIRE }}^{+}$

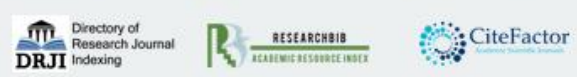

